\title{
Transient subdiffusion via disordered quantum walks
}

\author{
Andrea Geraldi $\odot,{ }^{1, *},{ }^{*}$ Syamsundar De $\odot,{ }^{2,},{ }^{*},+$ Alessandro Laneve, ${ }^{1}$ Sonja Barkhofen, ${ }^{2}$ Jan Sperling, ${ }^{2}$ \\ Paolo Mataloni, ${ }^{1}$ and Christine Silberhorn ${ }^{2}$ \\ ${ }^{1}$ Dipartimento di Fisica, Sapienza Università di Roma, Piazzale Aldo Moro, 5, 00185 Roma, Italy \\ ${ }^{2}$ Integrated Quantum Optics Group, Applied Physics, Paderborn University, 33098 Paderborn, Germany
}

(Received 24 July 2020; accepted 23 March 2021; published 16 April 2021)

\begin{abstract}
Transport phenomena play a crucial role in modern physics and applied sciences. Examples include the dissipation of energy across a large system, the distribution of quantum information in optical networks, and the timely modeling of spreading diseases. In this work we experimentally prove the feasibility of disordered quantum walks to realize a quantum simulator that is able to model general transient subdiffusive phenomena, exhibiting a sublinear spreading in space over time. Our experiment simulates such phenomena by means of a finely controlled insertion of various levels of disorder during the evolution of the walker, enabled by the unique flexibility of our setup. This allows us to explore the full range of subdiffusive behaviors, ranging from anomalous Anderson-like localization to normal diffusion for all experimentally accessible step numbers.
\end{abstract}

DOI: 10.1103/PhysRevResearch.3.023052

\section{INTRODUCTION}

Transport phenomena are ubiquitous in physics, often in connection with the prominent heat equation. Such phenomena are prime examples for normal diffusion processes in which the variance, quantifying the spatial spread of the system's distribution, increases linearly with time. Nevertheless, it is actually quite common to find natural processes whose short-term or long-term dynamics does not follow such a simple relation. Rather, these systems are characterized by a distribution that broadens according to a nonlinear power law [1,2], a behavior referred to as anomalous diffusion. In particular, a sublinear relation between variance and time, i.e., subdiffusion, is frequently observed in nature, such as in biological processes [3-6], wave propagation and scattering [7,8], the movement of charge carriers in amorphous semiconductors [9], disordered media [10], and many-body localization transitions [11]. Subdiffusion even applies to certain economic models [12].

Because of this vast range of applications and its fundamental importance, many attempts have been made during recent years to uncover the underlying physical mechanisms that lead to anomalous diffusion. Such theoretical models rely on a variety of physically motivated and more abstract approaches, such as fractal theory $[13,14]$, fractional Brownian motion [15], and continuous-time random walks [16,17]. Consequently, the possibility to simulate all kinds of anomalous

\footnotetext{
${ }^{*}$ These authors contributed equally to this work.

†andrea.geraldi@uniroma1.it

‡syamsundar.de@upb.de
}

Published by the American Physical Society under the terms of the Creative Commons Attribution 4.0 International license. Further distribution of this work must maintain attribution to the author(s) and the published article's title, journal citation, and DOI. diffusive behaviors in one experimental platform - and in a tunable manner - cannot only lead to significant insights into complex mathematical models but also enables us to study a plethora of processes in nature. Here we show that such a simulation task can, indeed, be realized by means of disordered quantum walks $(\mathrm{QWs})$.

QWs-the counterpart to classical random walks that exploit coherent superpositions-serve as a promising framework to implement simulation protocols since they provide a general model for the propagation of quantum particles [18-21]. For example, QWs have been used to study transport phenomena in biomolecules [22], evolution in solid-state systems [23], formation of molecular states [24], topological invariants [25,26], and edge states [27,28]. However, basic QWs are characterized by a spread which grows quadratically in time. This superdiffusive broadening is referred to as ballistic diffusion. Moreover, by actively influencing the walker's evolution, the functional dependency of the broadening can be altered, e.g., for reproducing the classical normal diffusion of incoherent processes, Anderson localization, or even hypertransport $[21,29,30]$. For instance, static disorder leads to Anderson localization, arising from the interaction between the coherent quantum walker and a disordered environment [31,32].

Recently, the continuous transition from ballistic behavior to normal diffusion has been experimentally demonstrated in a QW through the implementation of inhomogeneous evolution patterns, according to a so-called p-diluted model [33]. This approach proves that superdiffusion is achievable by introducing inhomogeneities, but it is incapable of implementing other anomalous diffusion regimes that result from the presence of disorder. Therefore, the less accessible entirety of the subdiffusive domain remains largely unexplored, even when restricting to the transient subdiffusive regimes as the true asymptotic behavior is experimentally inaccessible. Among other reasons, a lack of a fitting experimental platform 
hindered such an investigation to date since it has to be scalable, dynamically reconfigurable, and compatible to the introduction of controlled disorder in the spatial and temporal domain in order to realize advanced disorder models.

In this paper we experimentally demonstrate that the conceptual idea of $p$-diluted disorder can be critically extended to encompass the subdiffusive transient regime, i.e., diffusive behaviors for the maximally accessible number of time steps. In contrast to earlier implementations of the superdiffusive domain, our experiment uses a highly flexible time-multiplexing scheme to resolve the open problem of simulating subdiffusion processes. By controlling disorder in the spatial degree of freedom (here, time bins) and in time (here, number of steps), we show the possibility to realize any transient sublinear propagation regime-ranging from statically disordered QWs, giving birth to Anderson-like localization, to completely disordered QWs, corresponding to normal diffusion-that is achieved by implementing different disorder levels. Our experiment is supplemented by a theoretical model and numerical simulations, as well as a space-time-coin-resolved characterization of QWs.

\section{THEORETICAL MODEL}

A broadly applicable model for general transport processes can be formulated in terms of the partial differential equation

$$
0=\partial_{t} P(x, t)+\mathcal{L} P(x, t),
$$

in which $P(x, t)$ represents a space-time dependent probability distribution and $\mathcal{L}$ is a potentially time-dependent differential operator in the spatial degree of freedom $x$. For example, $\mathcal{L} \propto$ $-\partial_{x}^{2}$ describes the heat equation with normal diffusion. For a large family of randomized media, the asymptotic solution for large times $t$ reads

$$
P(x, t) \propto \exp \left(-\left|\frac{a x}{\sigma(t)}\right|^{b}\right),
$$

where $b$ describes the type of the exponential decay and $a$ is a scaling factor. Furthermore, $\sigma(t)$ is the standard deviation with the characteristic power-law behavior,

$$
\sigma(t)=c t^{d},
$$

where $2 d$ determines the spread of the variance over time and $c$ is another scaling factor. See, e.g., Ref. [34] for a thorough derivation of this model; here we apply this model as an heuristic approach to interpreting our data. For example, for $b=2$ and $2 d=1$ we get from the rigorous model a Gaussian distribution in space with a linear increase of the variance, while the parameters $b=1$ and $2 d=0$ result in Andersonlike localization as a consequence of the static disorder. Here we aim at exploring the theoretically predicted intermediate regime $1<b<2$, with a subdiffusive behavior $0<d<1 / 2$.

As established before, discrete QWs have shown their ability to simulate certain diffusion regimes, such as superdiffusive power laws [33], in the continuous limit of many steps and positions. The walker on a line is described by the coherent superposition state $|\psi(t)\rangle=\sum_{x}\left(\psi_{0}(x, t)|x\rangle \otimes\right.$ $\left.|0\rangle+\psi_{1}(x, t)|x\rangle \otimes|1\rangle\right)$, where $\{|0\rangle,|1\rangle\}$ represents the quantum coin. For the resulting probability distribution, we trace over this internal degree of freedom $P(x, t)=\left|\psi_{0}(x, t)\right|^{2}+$ $\left|\psi_{1}(x, t)\right|^{2}$. The QW evolves through the action of two operators, the coin operator $\hat{C}(t)$ and the step operator $\hat{S}$. The coherent coin toss is given by the unitary map $\hat{C}(t)=\sum_{x}|x\rangle\langle x| \otimes$ $\hat{C}(x, t)$, which can vary with positions and times. The step operator $\hat{S}=\sum_{x}(|x-1\rangle\langle x|\otimes| 0\rangle\langle 0|+| x+1\rangle\langle x|\otimes| 1\rangle\langle 1|)$, then coherently propagates the walker in the two directions, depending on the coin state. Thus, the evolution of the full quantum system reads $|\psi(t+1)\rangle=\hat{S} \hat{C}(t)|\psi(t)\rangle$.

It is convenient to model different anomalous diffusion regimes with a corresponding degree of static and dynamic disorder in the choice of the space-time dependent coin. The degree of the dynamic variation is determined by a parameter $p$, resulting in the notion of $p$-diluted disorder [33]. In general, the coin operator is not homogeneous with respect to position and step number and different constraints can be imposed [35]. For instance, the coin operator can be inhomogeneous in space, but static in time, $\hat{C}(x, t)=\hat{C}(x)$, leading to Andersonlike localization $(b=1)$, which is a static effect $(2 d=0)$. Now this static disorder can be perturbed in the $p$-diluted model to approximate different differential operators $\mathcal{L}$ in Eq. (1) for different physical scenarios. This perturbation consists of the independent and random choice of time-dependent coin configurations according to the percentage of dynamic disorder $p$,

$$
\hat{C}(x, t)= \begin{cases}\hat{C}(x, t) & \text { with probability } p, \\ \hat{C}(x) & \text { with probability } 1-p,\end{cases}
$$

which introduces an inhomogeneity in time. Specifically, $p=$ 0 yields Anderson-like localization ( $b=1$ and $2 d=0$ ), and $p=1$ results in a completely disordered QW $(b=2$ and $2 d=1$ ). Most importantly, the region $0<p<1$ should theoretically enable us to control our QW in such a way that it explores the full intermediate range of exponential spatial decays, $1<b<2$ in Eq. (2), with sublinear temporal spreads, $0<2 d<1$ in Eq. (3). See Appendix A for technical details and the connection to transport processes. It is worth mentioning that our $p$-diluted strategy applies to the transient regime of a limited step number in experiments and not to the rigorous asymptotic behavior, which often tends to saturate in a diffusive long-term propagation.

\section{EXPERIMENTAL IMPLEMENTATION}

To implement this transient subdiffusion, our QW experiment relies on the well-established time-multiplexing scheme based on an unbalanced Mach-Zehnder interferometer with a feedback loop [32,36,37]; see Fig. 1. Our scheme provides high resource efficiency, long-lasting stability, and homogeneity, which we exploit for the realization of QWs over a sufficiently large number of steps that is necessary for clearly distinguishing the signatures of the subdiffusive behavior. This is not possible with the experiment in Ref. [33] since it relies on a spatial geometry that is comparably hard to scale.

In the scheme in Fig. 1, the positions are encoded in the arrival time bin of a weak coherent laser pulse at the singlephoton level (central wavelength $1550 \mathrm{~nm}$, pulse width $1 \mathrm{ps}$, and repetition rate $4 \mathrm{kHz}$ ). This light acts as the walker while the coin information is embedded in the polarization, $|H\rangle=$ $|0\rangle$ and $|V\rangle=|1\rangle$. The walk starts when the pulse impinges from the top port of $\mathrm{PBS}_{1}$ for the first time. The walk is then 


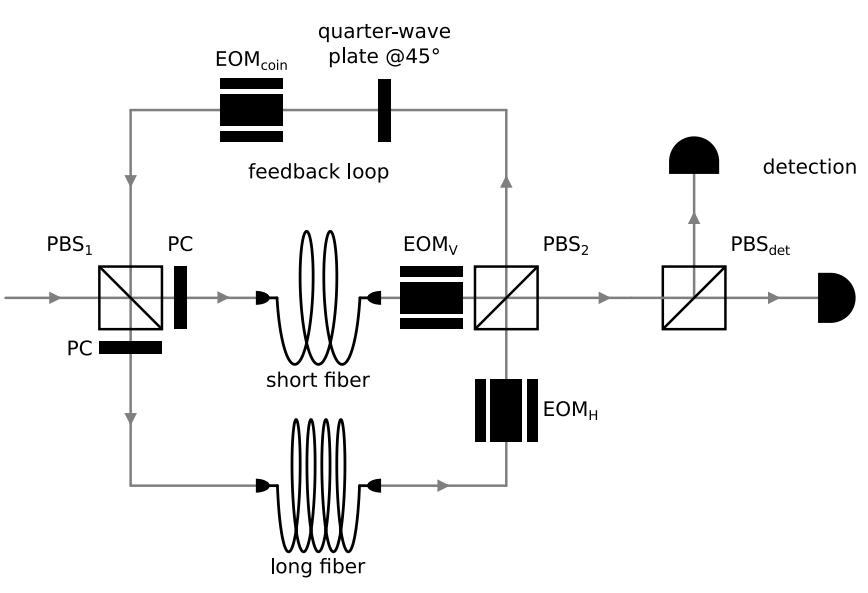

FIG. 1. Schematics of our experimental layout, using polarization controllers (PCs), polarizing beam splitters (PBSs), and electro-optical modulators (EOMs). The PC allows us to precisely compensate the polarization rotation caused by the propagation through the fibers. A polarization-resolving detection is achieved by splitting the output of the loop with $\mathrm{PBS}_{\text {det }}$ followed by one detector for each polarization.

initialized at position $x=0$ with horizontally polarized light $|\psi(0)\rangle=|0\rangle \otimes|0\rangle$.

An unbalanced interferometer with a well-defined delay between the polarizations (105 ns) realizes the step operation $\hat{S}$. This includes polarization dependent splitting at $\mathrm{PBS}_{1}$, propagation of horizontal and vertical polarization through long $(\sim 473 \mathrm{~m})$ and short $(\sim 453 \mathrm{~m})$ fibers, respectively, and the coherent recombination of the two paths at $\mathrm{PBS}_{2}$ to introduce the delay between the two polarizations. The interferometer is closed with a free-space feedback loop that redirects the light back to $\mathrm{PBS}_{1}$ for the next step.

A position- and step-dependent coin operation is harnessed for the implementation of $p$-diluted disorder that is central for realizing subdiffusive dynamics. To this aim, an essential step forward is the exploitation of a fast-switching EOM in the feedback loop for a dynamical coin control via polarization rotations without introducing high additional losses. See Appendix B for technical details of the coin operation.

The capability of dynamical polarization rotation by the two fast-switching EOMs, $\mathrm{EOM}_{\mathrm{H}}$ and $\mathrm{EOM}_{\mathrm{V}}$, enables us routing the pulses either back to the feedback loop or to the detection unit. This high-quality active polarization control facilitates deterministic in and out coupling, rendering it possible to implement sufficiently large number of steps by enhancing the round trip efficiency. The detection unit allows for polarization-resolved photon counting at individual time bins, using PBS $_{\text {det }}$ and high-efficiency (>90\%) superconducting nanowire single-photon detectors with a dead time of $\sim 100 \mathrm{~ns}$, from which we deduce the evolution of walker's probability distributions.

The current setup is designed to have a step separation of $\sim 2.3 \mu$ s and position separation of $\sim 105 \mathrm{~ns}$ and has been utilized to demonstrate walks up to 36 steps by allowing time-bin interlacing for successive steps [37]. However, we here restrict ourselves to 20 steps, which is sufficient to unambiguously discern transient subdiffusive dynamics, while minimizing the error from interlacing.

\section{RESULTS}

Our goal is to explore transient subdiffusive dynamics by studying the walker's behavior as a function of the disorder level.

For a given strength of disorder $p$, several coin configurations can be obtained because of the randomness in the choice of the coin [Eq. (4)]. We refer to each configuration as a coin map. Relevant quantities can be extracted from the output distribution $P(x, t)$ after averaging over many realizations for a given $p$. We experimentally implemented 400 coin maps for each disorder scenario under study,

$$
p \in\{0.0,0.1,0.2,0.3,0.5,1.0\},
$$

and the resulting average probability distribution has been measured for step numbers

$$
t \in\{5,8,11,14,17,20\} .
$$

The 2400 coin maps are generated by randomly creating initially statically disordered coin configurations; for that, it suffices to pick one coin value from three possible ones independently for each site of the line. We then divide the coin maps into six sets, each comprising 400 coin maps, and impose on each subset a different disorder level. This is achieved by altering the coin value at each position and time to another one, with a probability equal to $p$. The new coin values are chosen with uniform probability. This results in 400 different coin maps with the same disorder $p$. By randomly setting the initial static disorder for each coin map, it is assured that the final results do not depend on a particular static configuration but only on the disorder level $p$.

Our measured data enables us to analyze both the spatial characterization via $P$ with a given exponential behavior $1<$ $b<2$, and the temporal spread to certify anomalous diffusion $0<2 d<1$. Eventually we discuss the coin-resolution capabilities of our experiment.

\section{A. Spatial characterization}

For a fixed step number $t$, and for $p=0$, an exponentially localized distribution is expected, corresponding to Andersonlike localization. With increasing disorder level $p$, we expect a broadening of the distribution. Eventually, for $p=1$, a Gaussian shape should be obtained, being typical for the diffusive behavior. In order to find the parameters that fit the measured distribution best, it is convenient to work with a modified expression of Eq. (2),

$$
\ln (P)=\left(-\left|\frac{a}{\sigma}\right|^{b}\right)|x|^{b}-\ln \left(\sum_{x} e^{-|a x / \sigma|^{b}}\right),
$$

that can be fitted to our experimental data.

Experimental data corresponding to $t=20$ steps for different amounts of disorder are reported in the left column of Fig. 2. In the top-left plot, dots correspond to experimentally obtained probability distributions. Dotted lines represent values of the theoretical distribution, obtained from a numerical simulation. Similarities between experimental and theoretical 

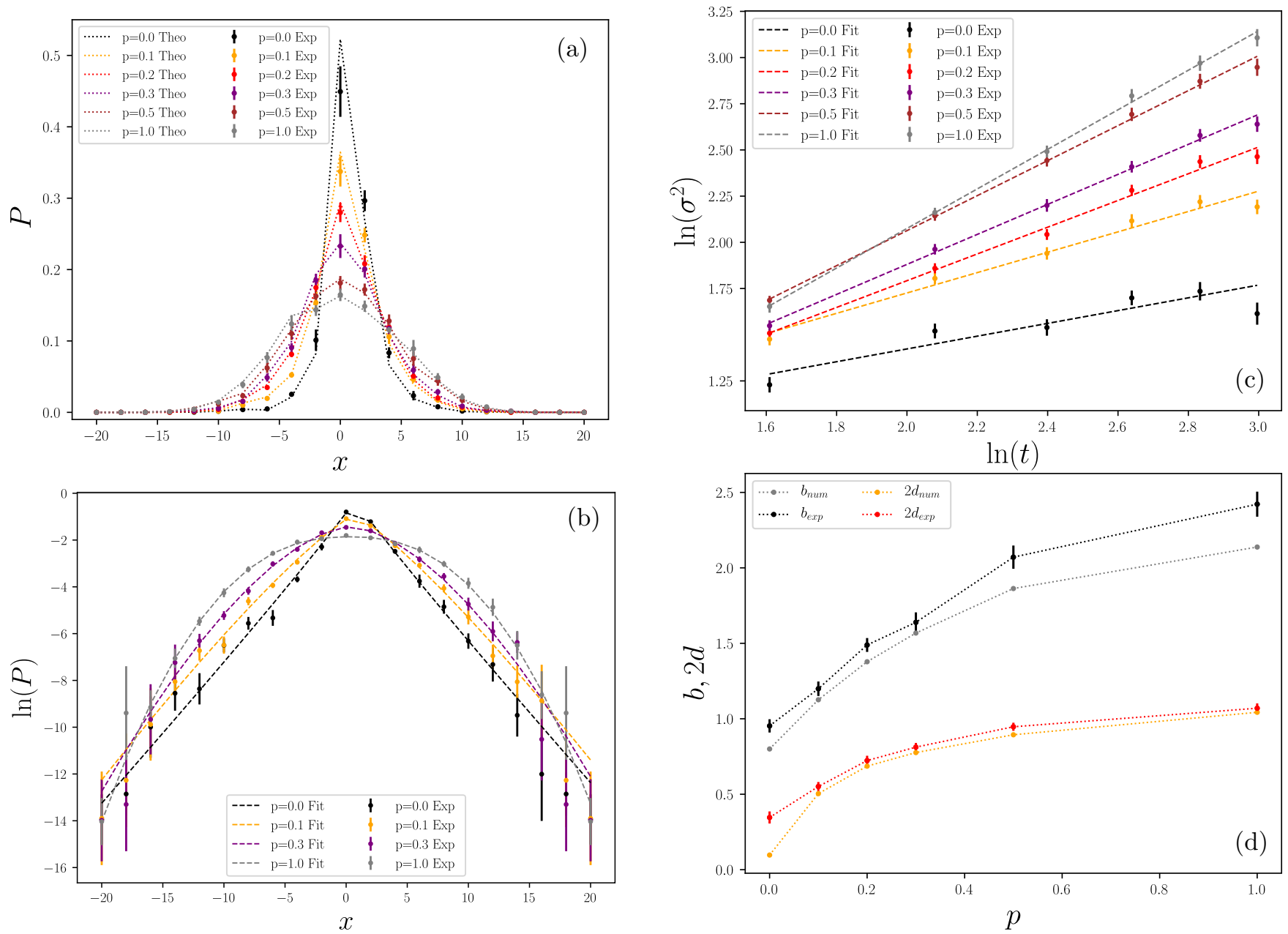

FIG. 2. Top left: Probability distribution $P$ for various values of the disorder $p$ [Eq. (5)]. Experimental data (dots) agree within the uncertainties with the theoretical results (dotted lines). Error bars take into account Poissonian statistics and experimental imperfections of the setup [26,38]. Bottom left: Logarithm of the experimental probability distribution (dots) together with fit (dashed lines) according to Eq. (7). For the sake of clarity, only selected $p$ values are depicted. Top right: Logarithm of the variance as a function of the logarithm of the step number $t$ [Eq. (6)] for disorder levels $p$ in Eq. (5). Dots correspond to experimental data; dashed lines show fits according to Eq. (8). The linear behavior with slopes between zero and one in this doubly logarithmic graph for each value of $p$ demonstrates an excellent agreement with the predicted subdiffusive nature of the evolution. Bottom right: Depiction of values of the exponents for the spatial $(b)$ and temporal $(2 d)$ characteristics, obtained by fitting the theoretical predictions in Eqs. (7) and (8) to the measured data in bottom-left and top-right plots.

distributions for each step and $p$ values are above $99 \%$, indicating a very good agreement even without considering many unavoidable experimental imperfections in our simulation.

The bottom-left panel of Fig. 2 is even more conclusive when it comes to determining the characteristic exponent $b$. The plot shows data (dots) together with the fitted curves (dashed lines) according to Eq. (7), covering the range from a linear $(b \approx 1)$ to a parabolic $(b \approx 2)$ decay in this logarithmic depiction. It is clear from the graph that the presence of higher disorder $p$ diminishes the probability to find the walker in the starting position $x=0$ for $t>0$. Consequently, the probability for more distant positions increases, resulting in a broadened distribution. The transient subdiffusivity of the evolution is confirmed through $1 \lesssim b \lesssim 2$ (the specific numerical values are reported in the bottom-left plot of Fig. 2). It is worth noting that other imperfections lead to a broader range than one actually expects from an ideal model, cf. value $b>2$.

\section{B. Temporal characterization}

The second feature we focus on consists of the dependency of the variance as a function of the step number $t$, again for different values of disorder. To assess the transient subdiffusive spread with our data, it is similarly convenient to recast Eq. (3) into a logarithmic form,

$$
\ln \left(\sigma^{2}\right)=2 d \ln (t)+\ln \left(c^{2}\right) .
$$

Results are reported in the top-right plot of Fig. 2 on a logarithmic scale for both axes. Dashed lines correspond to the curve in Eq. (8) which is fitted to the data (dots) for different $p$ values. The nearly perfect linear behavior with slopes $0 \lesssim$ $2 d \lesssim 1$ (see bottom-right depiction in Fig. 2 for the values and Appendix $\mathrm{C}$ for further details) confirms the actual transient subdiffusive spread of the QW evolution. A discrepancy between the data and the fit can be observed for $p=0.0$ because of the extreme sensitivity of Anderson-like localization with 


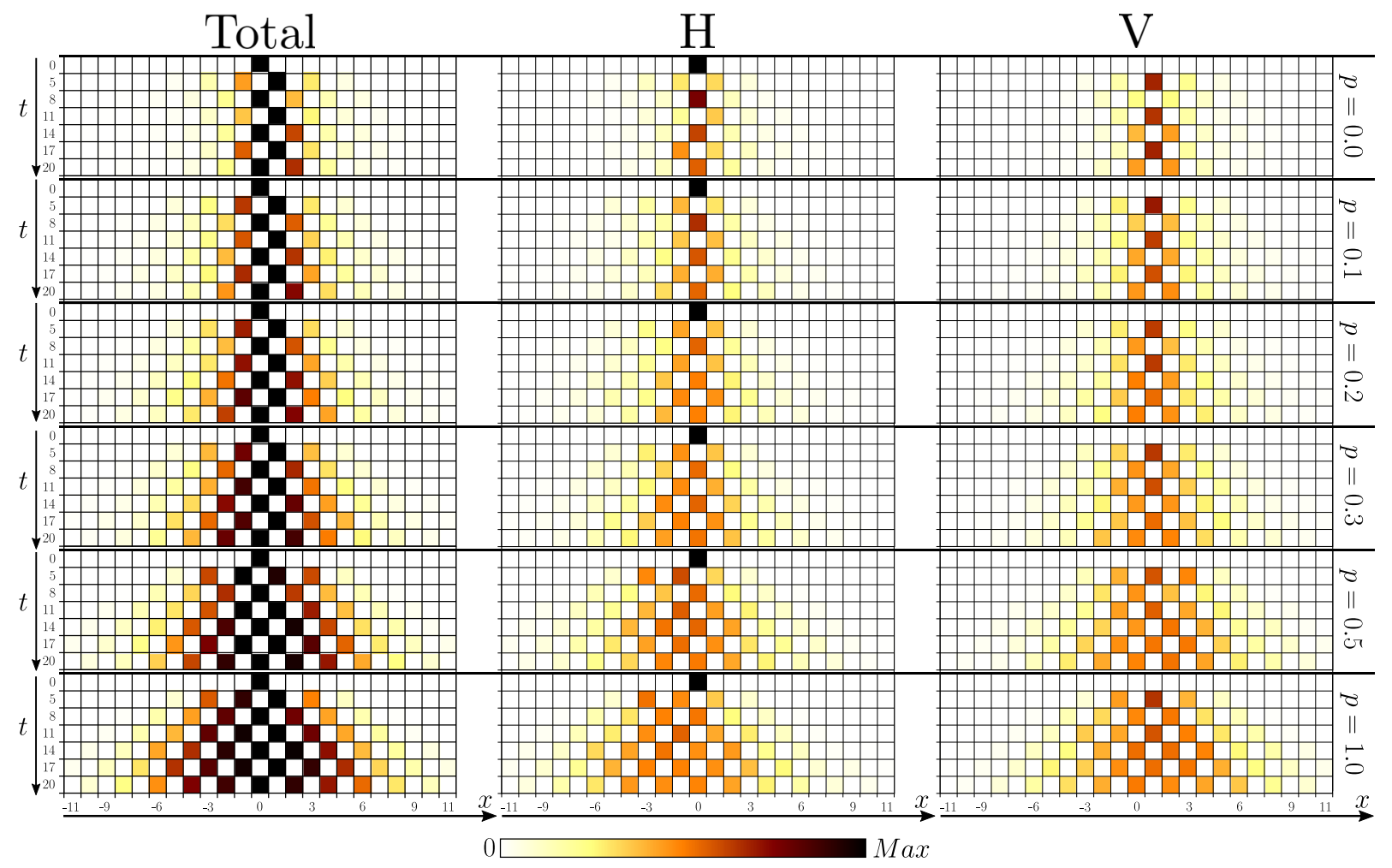

FIG. 3. Coin-resolved time evolution of the walkers position-dependent distribution for all disorder values $p$ under investigation [Eq. (5)]. Left column corresponds to the overall space-time behavior after tracing over the coin degree of freedom while the central and right column show the cases that correspond to the coin states $|0\rangle$ and $|1\rangle$, respectively. The depicted data include all measured time steps $t$ [Eq. (6)] and all positions $x \in[-11,11]$. For an enhanced visualization, each row of the total distribution is normalized to its maximum. Each row of the $H$ and $V$ plots are normalized to the maximum of the corresponding row of the plot labeled as "Total."

respect to unavoidable experimental imperfections. Here one would also expect a constant variance, which, however, is only approached in the limit $t \rightarrow \infty$, even in theory. As in the previous spatial analysis, error bars on the experimental data have been computed considering a Poissonian statistic of counting as well as experimental imperfections.

Beyond earlier studies, we analyzed both the spatial and temporal impact of the amount of disorder $p$. According to our results, we can confirm that our approach enables us to simulate almost any subdiffusive behaviors, within the natural restrictions of a finite number of steps in experiments. Thus, the transient sublinear spread over time and the characteristic shapes of the measured spatial distributions indicate that the interplay between a static disorder and completely random disorder, freely controlled and interpolated via $p$, is a viable way to reproduce complex subdiffusion phenomena in discrete QWs.

\section{Full space-time-coin analysis}

In addition to the study of the walker's spread over the spatial sites of the discretized line, our actual setup allows one to study the polarization-resolved evolution of the walker. In this way, one can follow the average behavior of the particle by decoupling the two coin states.

Measurements corresponding to the evolution of the $|0\rangle=$ $|H\rangle$ (center) and $|1\rangle=|V\rangle$ (right) coin states are reported in Fig. 3. The left plot shows the total space-time-dependent characterization of the QW after tracing over the coin degree of freedom. Each column represents the time behavior of the probability distribution as a function of the site (from site -11 to +11 ) for different values $p$ of the disorder.

At step $t=0$, the coin state has 0 probability to be in the $|1\rangle_{c}$ state as the walker is initially furnished with a coin in the state $|0\rangle_{c}$. Note that the distribution for $|0\rangle$ is centered in site -1 while the one for $|1\rangle$ is centered in site +1 . This is due to the effect of the step operator $\hat{S}$, which shifts the walker with coin $|0\rangle$ and $|1\rangle$ to the left and right, respectively, thus steering the coin distribution towards different signs of sites. This effect is compensated when tracing over the polarization degree of freedom. Despite this, an under diffusive spread for both coin states is observed, which is very similar to the one obtained when tracing over the coin degree of freedom. Indeed, Anderson-like localization is obtained in the case of $p=0.0$, while a diffusive (i.e., a Gaussian distribution) spread is retrieved in the case of complete disorder. For all values of $p<1.0$, a more narrowly confined spread with respect to $p=1.0$ is obtained.

Therefore, and in addition to the vital characterization of spatial and temporal features of various QWs, we can also investigate the coin-dependent transient behavior with our setup. Here, such a fine-grained analysis resembles the overall properties of the system. However, in other scenarios, this additional degree of resolution can be a helpful asset to separate the distinct characteristics of the system when conditioned to a given coin state. 


\section{CONCLUSION}

In pursuing the ultimate goal of implementing a universal quantum simulator, we experimentally demonstrated the ability to simulate transient subdiffusive transport phenomena, having a wide range of applications, via disordered QWs. By analyzing our data regarding their spatial and temporal features, we mapped the landscape of characteristic properties of subdiffusion. First, we controlled our system in a way that leads to position distributions of the walker ranging from Anderson-like localization to a normal Gaussian distribution. Second, anomalous diffusion in the sublinear regime was explored to characterize the walker's spread over a finite time. This complements earlier findings that have been restricted to superdiffusion by starting from an already completely ordered evolution.

Because of our unique control over the coin at each position (time bin) and step, the demanding goal of realizing subdiffusion was successfully accomplished. By perturbing initial static disorder, we realized a $p$-diluted QW by adding dynamic noise in a controlled manner to steer our system towards the subdiffusive regime.

The agreement between the measured data and the theoretical predictions for the key quantities under study, namely shape of the position distribution and the change of the variance with time, clearly demonstrates that the coherent walker evolves subdiffusively.

Earlier works that implied the possibility of subdiffusive dynamics in QWs invoked the presence of nonlinearities in the coin operator $[39,40]$. Such approaches generally involves setups with high complexity and low efficiency [41], in addition to the more challenging theoretical treatment. In contrast, we were able to realize subdiffusive QWs in a significantly more accessible manner, providing an efficient tool for the investigation of anomalous diffusion in the transient regime. This has been done by implementing a crossover between two disorder models: a static one, leading to Anderson-like localization, and the dynamical one, inducing diffusion. Through a continuous transition from one model to the other, straightforwardly encoded in the increment of a single parameter $(p)$, the entirety of the subdiffusive regime can be explored.

Exceeding our proof-of-concept realization reported here, our results provide a promising starting point for future studies. For instance, we also showed that our setup actually enables us to measure coin-resolved distributions that can be relevant for assessing quantum properties between the coin and time-bin degrees of freedom, such as entanglement. Furthermore, the experiment could be extended to two singlephoton walkers [33] by means of the very same setup [42]. This could foster other simulations of sophisticated correlated diffusion phenomena. For instance, it is known that Anderson localization holds true in the case of entangled photons [31]. However, the general impact of correlated $\left(p_{1}, p_{2}\right)$-diluted dynamical noise for the walkers 1 and 2 is entirely unknown but could potentially be studied with our platform. In addition to this, our $p$-diluted disorder approach achieves the fundamental goal of reproducing different spreading behaviors in a single, stable, and reconfigurable setup. As such, it can serve to understand propagation properties of quantum particles in more complicated networks subjected, for instance, to space-, time-, and coin-dependent environmental noise contributions.

\section{ACKNOWLEDGMENTS}

The Integrated Quantum Optics group acknowledges financial support through the Gottfried Wilhelm Leibniz-Preis (Grant No. SI1115/3-1) and the European Commission through the ERC project QuPoPCoRN (Grant No. 725366). A.G., A.L., and P.M. acknowledge support from the European Commission Grants FP7-ICT-2011-9-600838 (QWAD - Quantum Waveguides Application and Development) and from the project PRIN 2017 'Taming complexity via QUantum Strategies a Hybrid Integrated Photonic approach' (QUSHIP), Id. 2017SRNBRK

\section{APPENDIX A: SUPPLEMENTAL DETAILS ON THE THEORY}

For a self-consistent reading of this work and for a coherent treatment, we recapitulate and reformulate the theory on diffusion in randomized media as reported in Ref. [34]. Therein, the approach was based on the Laplace transform in the temporal domain. For our purposes, it is more convenient to discuss that method in terms of the Fourier transform in the spatial domain. Eventually we relate this approach to $p$ diluted models.

A general model of diffusion in a one-dimensional system can be described by the equation

$$
0=\partial_{t} P(x, t)+\mathcal{L}\left(-\partial_{x}^{2}, t\right) P(x, t),
$$

where $\mathcal{L}$ is a potentially time-dependent differential operator. In the continuous limit, this equation also models the asymptotic behavior of a discrete system, such as ours. Furthermore, the differential operator depends on $-\partial_{x}^{2}$ for a positive (i.e., dispersive) behavior because of $-\partial_{x}^{2} e^{i x k}=k^{2} e^{i x k}$ and $k^{2} \geqslant 0$.

Using the characteristic function, i.e., the Fourier transform $\Phi(k, t)=\int_{-\infty}^{+\infty} d x e^{-i k x} P(x, t)$, we can rewrite Eq. (A1) as

$$
0=\partial_{t} \Phi(k, t)+\mathcal{L}\left(k^{2}, t\right) \Phi(k, t) .
$$

Then the solution in form of the Green's function can be formally expressed as

$$
\tilde{G}(k, t)=\exp \left(-\int_{0}^{t} d t^{\prime} \mathcal{L}\left(k^{2}, t^{\prime}\right)\right) .
$$

This solves Eq. (A2) as $\Phi(k, t)=\tilde{G}(k, t) \Phi(k, 0)$, where $\Phi(k, 0)$ represents the initial distribution. In our case, this is modeled by a singular input at the center position, thus $\Phi(k, 0)=1$ in the Fourier domain.

It was also shown in Ref. [34] that, for large times $(t \gg 1)$, solutions follow the functional form:

$$
P(x, t)=\frac{a b}{2 \sigma(t) \Gamma(1 / b)} \exp \left(-\left|\frac{a x}{\sigma(t)}\right|^{b}\right),
$$

with $\Gamma$ being the Gamma function and $\sigma(t)$ denoting a timedependent standard deviation. In addition, we define $a=$ $\sqrt{\Gamma(3 / b) / \Gamma(1 / b)}$ and $b$ relates to the type of exponential decay; e.g., $b=1$ and $b=2$ define a linear and quadratic behavior, respectively. 
The moments of this distribution can be evaluated as well; odd moments vanish and even moments read

$$
E\left(x^{2 n}\right)=\frac{\Gamma\left(\frac{2 n+1}{b}\right)}{\Gamma\left(\frac{1}{b}\right)}\left[\frac{\sigma(t)}{a}\right]^{2 n} .
$$

These moments enable us to expand the characteristic function in a Taylor series as

$$
\begin{aligned}
\Phi(k, t) & =\sum_{n=0}^{\infty} E\left(x^{n}\right) \frac{[i k]^{n}}{n !} \\
& =1+\sigma(t)^{2} \frac{-k^{2}}{2}+\frac{\Gamma\left(\frac{5}{b}\right) \Gamma\left(\frac{1}{b}\right)}{\Gamma\left(\frac{3}{b}\right)^{2}} \sigma(t)^{4} \frac{k^{4}}{24}+\cdots .
\end{aligned}
$$

Similarly, we can expand the generator of the evolution $\mathcal{L}\left(k^{2}, t\right)=\sum_{n=0}^{\infty} \lambda_{2 n}(t) \frac{k^{2 n}}{(2 n) !}$. This further allows us to expand the Green's function from Eq. (A3),

$$
\begin{aligned}
& \tilde{G}(k, t) \\
& =\tilde{G}(0, t)+\left[-\int_{0}^{t} d t^{\prime} \lambda_{2}\left(t^{\prime}\right)\right] \tilde{G}(0, t) \frac{k^{2}}{2} \\
& +\left\{-\int_{0}^{t} d t^{\prime} \lambda_{4}\left(t^{\prime}\right)+3\left[\int_{0}^{t} d t^{\prime} \lambda_{2}\left(t^{\prime}\right)\right]^{2}\right\} \tilde{G}(0, t) \frac{k^{4}}{24}, \\
& +\cdots,
\end{aligned}
$$

where $\tilde{G}(0, t)=\exp \left(\int_{0}^{t} d t^{\prime} \lambda_{0}\left(t^{\prime}\right)\right)$. Because of our initial conditions, resulting in $\Phi(k, t)=\tilde{G}(k, t)$, we can now equate the coefficients for $\widetilde{G}$ in Eq. (A7) and $\Phi$ in Eq. (A6). Since this identification has to be satisfied for all times $t>0$, we find

$$
\begin{aligned}
\lambda_{0}(t) & =0, \quad \int_{0}^{t} d t^{\prime} \lambda_{2}\left(t^{\prime}\right)=\sigma(t)^{2}, \quad \text { and } \\
-\int_{0}^{t} d t^{\prime} \lambda_{4}\left(t^{\prime}\right) & =\underbrace{}_{\stackrel{\text { def. }}{=}=\underbrace{\left[\frac{\Gamma\left(\frac{5}{b}\right) \Gamma\left(\frac{1}{b}\right)}{\Gamma\left(\frac{3}{b}\right)^{2}}-3\right]}_{\varphi=f(b)} \sigma(t)^{4} .}
\end{aligned}
$$

Importantly, $b=f^{-1}(\varphi)$ determines the exponent in Eq. (A4). See Fig. 4 for the graph of $f$.

A first consequence of the aforementioned relations is that the spread $\sigma(t)$ is given by the time dependency of the first nonzero Taylor coefficient $\lambda_{2}(t)$, resulting the the corresponding power law, such as $\sigma(t)=c t^{d}$ with constants $c, d>0$ [34]. Thus, the introduction dynamic disorder, changing the time-dependence generator $\mathcal{L}$, generally results in an increment of the power. As a second observation, we have a look at the relation that includes $\lambda_{4}(t)$. This coefficient is typically negative which allows one to substitute it by $-\lambda_{4}(t)=$ $\rho(t) \lambda_{2}(t)^{2}$. With this, we can rewrite the above relation as

$$
\begin{aligned}
\varphi= & \frac{\int_{0}^{t} d t^{\prime} \rho\left(t^{\prime}\right) \lambda_{2}\left(t^{\prime}\right)^{2}-\left(\int_{0}^{t} d t^{\prime} \rho\left(t^{\prime}\right) \lambda_{2}\left(t^{\prime}\right)\right)^{2}}{\left(\int_{0}^{t} d t^{\prime} \lambda_{2}\left(t^{\prime}\right)\right)^{2}} \\
& +\left(\frac{\int_{0}^{t} d t^{\prime} \rho\left(t^{\prime}\right) \lambda_{2}\left(t^{\prime}\right)}{\int_{0}^{t} d t^{\prime} \lambda_{2}\left(t^{\prime}\right)}\right)^{2} .
\end{aligned}
$$

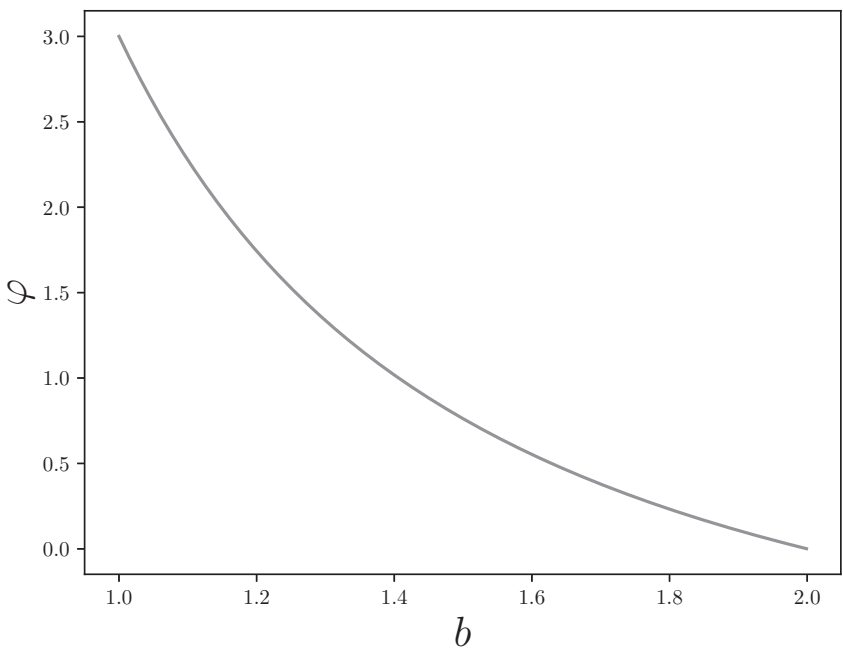

FIG. 4. Function $\varphi=f(b)=\Gamma\left(\frac{5}{b}\right) \Gamma\left(\frac{1}{b}\right) / \Gamma\left(\frac{3}{b}\right)^{2}-3$ is shown in the relevant interval $1 \leqslant b \leqslant 2$. In this region, $f$ is strictly monotonously decreasing, allowing for defining its inverse for determining $b$ from $\varphi$.

Herein the numerator of the first term plays a role a variance, quantifying the fluctuation in $\lambda_{2}(t)$, that influences $b=f^{-1}(\varphi)$.

With these considerations, we can conclude that our $p$-diluted model dynamically changes the generator $\mathcal{L}$ by altering the coin operations. As discussed above, this broadens the spread in time (increasing $d$ via $\lambda_{2}$ ). Second, it changes the spatial exponential decay. That is, if only a few coins are changed per time step (low $p$ ), those are unlikely the same coins, leading to a high fluctuation in $\lambda_{4}$, thus high $\varphi$, thus low $b$ (Fig. 4). The other way around, a high $p$ results in low $\varphi$ and a high $b$.

\section{APPENDIX B: EXPERIMENTAL COIN CONTROL}

Here we present details of the operation of our timemultiplexing QW setup based on a fiber loop as shown in Fig. 1. See also Refs. [32,36,37]. This scheme is beneficial in terms of resource efficiency, high stability, and high homogeneity, which we exploit to realize coherent evolution over sufficiently large number of steps.

Our investigation of subdiffusive behavior mainly relies on the implementation of position and step dependent coin operation $\hat{C}(x, t)$. This dynamical coin operation is achieved by extending the capability of the previous setup via the introduction of another fast-switching EOM $\left(\mathrm{EOM}_{\mathrm{coin}}\right)$ followed by a quarter-wave plate (QWP) in the feedback path. The action of a QWP aligned at an angle $45^{\circ}$ with respect to the polarization basis $\{|H\rangle,|V\rangle\}$ reads as

$$
\hat{C}_{\mathrm{QWP}}=\frac{1}{\sqrt{2}}\left(\begin{array}{cc}
1 & -i \\
-i & 1
\end{array}\right) .
$$

The EOM operation can be written as

$$
\hat{C}_{\mathrm{EOM}}=\left(\begin{array}{cc}
\cos \phi & -i \sin \phi \\
-i \sin \phi & \cos \phi
\end{array}\right),
$$


TABLE I. Characteristic parameters for the implemented disorder levels $p$. The values have been estimated by means of a least square fit to theoretical predictions. Quantities with the subscript "num" have been extracted from our numerical model, considering 10000 coin maps. The subscript "exp" indicates quantities obtained from our data, realizing 400 coin maps. The parameters $b$ and $\delta$ relate to the theoretical prediction $\ln (P)=-\delta|x|^{b}+$ const. (where $\delta=|a / \sigma|^{b}$ when compared to main text) for the 20th step. Values for $2 d$ and $c^{2}$ are a result of the fit to the theoretical prediction $\ln \left(\sigma^{2}\right)=2 d \ln (t)+\ln \left(c^{2}\right)$.

\begin{tabular}{lcccccccc}
\hline \hline$p$ & $b_{\text {num }}$ & $b_{\exp }$ & $\delta_{\text {num }}$ & $\delta_{\exp }$ & $2 d_{\text {num }}$ & $2 d_{\text {exp }}$ & $c_{\text {num }}^{2}$ & $c_{\text {exp }}^{2}$ \\
\hline 0.0 & 0.800 & $0.953 \pm 0.044$ & 1.027 & $0.719 \pm 0.084$ & 0.097 & $0.346 \pm 0.040$ & 3.56 & $2.08 \pm 0.19$ \\
0.1 & 1.126 & $1.199 \pm 0.048$ & 0.367 & $0.300 \pm 0.034$ & 0.504 & $0.551 \pm 0.030$ & 1.88 & $1.87 \pm 0.14$ \\
0.2 & 1.378 & $1.489 \pm 0.046$ & 0.171 & $0.130 \pm 0.015$ & 0.686 & $0.723 \pm 0.032$ & 1.44 & $1.41 \pm 0.11$ \\
0.3 & 1.568 & $1.639 \pm 0.066$ & 0.095 & $0.081 \pm 0.013$ & 0.776 & $0.812 \pm 0.028$ & 1.376 & $1.293 \pm 0.087$ \\
0.5 & 1.863 & $2.071 \pm 0.077$ & 0.038 & $0.022 \pm 0.004$ & 0.894 & $0.947 \pm 0.027$ & 1.232 & $1.183 \pm 0.073$ \\
1.0 & 2.138 & $2.422 \pm 0.083$ & 0.016 & $0.008 \pm 0.002$ & 1.043 & $1.070 \pm 0.032$ & 0.967 & $0.937 \pm 0.070$ \\
\hline \hline
\end{tabular}

where the phase $\phi$ can be tuned by varying the voltage applied to the EOM. Their combination leads to the transformation

$$
\hat{C}_{\mathrm{EOM}} \hat{C}_{\mathrm{QWP}}=\left(\begin{array}{cc}
\cos \theta & -i \sin \theta \\
-i \sin \theta & \cos \theta
\end{array}\right) \text {, }
$$

using

$$
\theta=\phi+\frac{\pi}{4}
$$

and the identities $(\cos \phi-\sin \phi) / \sqrt{2}=\cos \theta$ and $(\cos \phi+$ $\sin \phi) / \sqrt{2}=\sin \theta$.

It is worth emphasizing that the present scheme utilizes free-space EOMs, which introduce very low losses $(<1 \%)$. The combination of active in and out coupling and freespace EOMs lead to a significantly improved round trip efficiency $(>80 \%)$ in comparison to the previous disordered time-multiplexing QW setup that employed integrated EOMs [32]. However, relatively high-voltage requirements for freespace EOMs comes with the hardware limitations that allow only three different voltage settings, $v \in\left\{-v_{1}, 0,+v_{1}\right\}$, during a single experimental run. In particular, $v=0$ corresponds to $\phi=0$, leading to a coin operation that equally mixes $|H\rangle$ and $|V\rangle$. We chose $v= \pm v_{1}$ such that $\phi=\mp \pi / 4$. This yields an identity coin that leaves the polarization states unchanged and a reflection coin that switches the polarizations. Notably, we find that these three accessible coin operations are sufficient for the exploration of the complete subdiffusive QW regime, thanks to the $p$-diluted disorder scheme. We design appropriate voltage-switching patterns for the EOMs that put into effect various disorder strengths $p$ ranging from $p=0$ (Anderson-like localization) to $p=1$ (normal diffusion).

\section{APPENDIX C: ADDITIONAL RESULTS FROM DATA ANALYSIS AND COMPARISON WITH NUMERICAL MODEL}

In order to compare our experimental results with the expected ones, we implemented a numerical simulation that produces 10000 different coin maps for a given level of disorder. Let us recall that a coin map is a set of coin configurations which are obtained by starting from static disorder $\hat{C}(x)$ and randomly changing a percentage $p$ of coins to $\hat{C}(x, t)$. The distribution that is obtained by averaging over all numerically implemented coin maps then models our experiment. However, these theoretical values have been computed in the completely ideal case, i.e., without considering unavoidable setup imperfections. Still, this simple model was already sufficient to match the results of the experiment sufficiently well.

The comparison to theory of both our data and numerical model are given in Table I for various cases of $p$-diluted disorder. These values follow the expected trend: the higher the disorder, the higher the exponential decay in space and temporal dispersion, quantified by $b$ and $d$, respectively. Specifically, the reported values confirm that we mostly operate in the subdiffusive regime of the $\mathrm{QW}, 1 \leqslant b \leqslant 2$ and $0 \leqslant 2 d \leqslant 1$. In addition to the logarithmic plot of the time-dependent variance in the main text, a linearly scaled version is provided in Fig. 5.

As one might expect, small discrepancies can be observed between experimental and numerical values as well as the theoretical predictions in Table I. For instance, deviations from numerical and experimental parameters can be caused by imperfect randomization since these parameters have been extracted by averaging the probability distributions over 10000 coin maps in the ideal simulation while only 400 have been implemented experimentally. Nevertheless, estimates for parameters from the numerics and data mostly agree with each other within the confidence interval, and deviations can be generally explained by considering experimental imperfections, such as a nonideal operation of the EOMs as well as

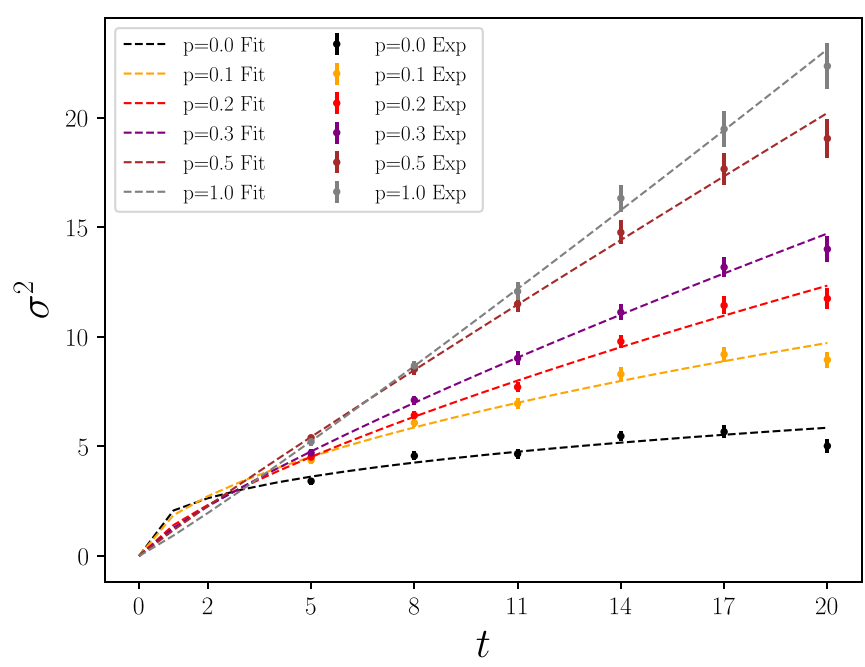

FIG. 5. Experimental data (dots) and the fitted relation $\sigma^{2}=$ $c^{2} t^{2 d}$ (dashed lines) on a linear scale for both axes. 
the QWP, i.e., a less than $100 \%$ visibility of interference, and slight setup misalignment, all of which contribute to increasing the spread of the walker.

The highest discrepancies to the theory can be observed for the extremal cases of disorder, $p=0$ and $p=1$, affecting both numerics and experiment. First, the discrepancy for $p \rightarrow$ 0 can be understood by considering that Anderson localization typically arises from a strict periodicity in the disorder pattern. For this reason, it is much more sensitive to small imperfec- tions compared to other disorder values, resulting in an higher deviation from the theory. Moreover, a nonspreading regime is only feasible for $t \rightarrow \infty$. Second, the discrepancy for $p \rightarrow 1$ is amplified by some of the effects previously mentioned even further. For instance, $p$-diluted models describe a convolution of the initial (Anderson-like) behavior with another distribution for dynamic disorder, causing that imperfections of all initial realizations add up. Besides, imperfections propagate along with the spreading of the walker over time.
[1] R. Metzler and J. Klafter, The restaurant at the end of the random walk: Recent developments in the description of anomalous transport by fractional dynamics, J. Phys. A: Math. Gen. 37, R161 (2004).

[2] R. Metzler, J.-H. Jeon, A. G. Cherstvy, and E. Barkai, Anomalous diffusion models and their properties: Non-stationarity, non-ergodicity, and ageing at the centenary of single particle tracking, Phys. Chem. Chem. Phys. 16, 24128 (2014).

[3] I. Bronstein, Y. Israel, E. Kepten, S. Mai, Y. Shav-Tal, E. Barkai, and Y. Garini, Transient Anomalous Diffusion of Telomeres in the Nucleus of Mammalian Cells, Phys. Rev. Lett. 103, 018102 (2009).

[4] H. L. Coker, M. R. Cheetham, D. R. Kattnig, Y. J. Wang, S. Garcia-Manyes, and M. I. Wallace, Controlling anomalous diffusion in lipid membranes, Biophys. J. 116, 1085 (2019).

[5] B. M. Regner, D. Vučinić, C. Domnisoru, T. M. Bartol, M. W. Hetzer, D. M. Tartakovsky, and T. J. Sejnowski, Anomalous diffusion of single particles in cytoplasm, Biophys. J. 104, 1652 (2013).

[6] Y. Golan and E. Sherman, Resolving mixed mechanisms of protein subdiffusion at the $\mathrm{t}$ cell plasma membrane, Nat. Commun. 8, 15851 (2017).

[7] S. Marano and M. Franceschetti, Ray propagation in a random lattice: A maximum entropy, anomalous diffusion process, IEEE Trans. Antennas Propag. 53, 1888 (2005).

[8] M. S. Toivonen, O. D. Onelli, G. Jacucci, V. Lovikka, O. J. Rojas, O. Ikkala, and S. Vignolini, Anomalous-diffusionassisted brightness in white cellulose nanofibril membranes, Adv. Mater. 30, 1704050 (2018).

[9] H. Scher and E. W. Montroll, Anomalous transit-time dispersion in amorphous solids, Phys. Rev. B 12, 2455 (1975).

[10] S. Havlin and D. Ben-Avraham, Diffusion in disordered media, Adv. Phys. 51, 187 (2002).

[11] K. Agarwal, S. Gopalakrishnan, M. Knap, M. Müller, and E. Demler, Anomalous Diffusion and Griffiths Effects Near the Many-Body Localization Transition, Phys. Rev. Lett. 114, 160401 (2015).

[12] J. Masoliver, M. Montero, and G. H. Weiss, Continuous-time random-walk model for financial distributions, Phys. Rev. E 67, 021112 (2003).

[13] W. Chen, H. Sun, X. Zhang, and D. Korošak, Anomalous diffusion modeling by fractal and fractional derivatives, Comput. Math. Appl. 59, 1754 (2010).

[14] D. Ben-Avraham and S. Havlin, Diffusion and Reactions in Fractals and Disordered Systems (Cambridge University Press, Cambridge, 2000).
[15] J.-H. Jeon and R. Metzler, Fractional Brownian motion and motion governed by the fractional Langevin equation in confined geometries, Phys. Rev. E 81, 021103 (2010).

[16] E. W. Montroll and G. H. Weiss, Random walks on lattices. II, J. Math. Phys. 6, 167 (1965).

[17] E. Abad, S. B. Yuste, and K. Lindenberg, Reaction-subdiffusion and reaction-superdiffusion equations for evanescent particles performing continuous-time random walks, Phys. Rev. E 81, 031115 (2010).

[18] Y. Aharonov, L. Davidovich, and N. Zagury, Quantum random walks, Phys. Rev. A 48, 1687 (1993).

[19] S. E. Venegas-Andraca, Quantum walks: A comprehensive review, Quant. Info. Proc. 11, 1015 (2012).

[20] J. Kempe, Quantum random walks: An introductory overview, Contemp. Phys. 44, 307 (2003).

[21] N. C. Harris, G. R. Steinbrecher, M. Prabhu, Y. Lahini, J. Mower, D. Bunandar, C. Chen, F. N. Wong, T. Baehr-Jones, M. Hochberg et al., Quantum transport simulations in a programmable nanophotonic processor, Nat. Photonics 11, 447 (2017).

[22] M. B. Plenio and S. F. Huelga, Dephasing-assisted transport: Quantum networks and biomolecules, New J. Phys. 10, 113019 (2008).

[23] M. Christandl, N. Datta, A. Ekert, and A. J. Landahl, Perfect State Transfer in Quantum Spin Networks, Phys. Rev. Lett. 92, 187902 (2004).

[24] A. Ahlbrecht, A. Alberti, D. Meschede, V. B. Scholz, A. H. Werner, and R. F. Werner, Molecular binding in interacting quantum walks, New J. Phys. 14, 073050 (2012).

[25] V. V. Ramasesh, E. Flurin, M. Rudner, I. Siddiqi, and N. Y. Yao, Direct Probe of Topological Invariants Using Bloch Oscillating Quantum Walks, Phys. Rev. Lett. 118, 130501 (2017).

[26] S. Barkhofen, T. Nitsche, F. Elster, L. Lorz, A. Gábris, I. Jex, and C. Silberhorn, Measuring topological invariants in disordered discrete-time quantum walks, Phys. Rev. A 96, 033846 (2017).

[27] L. Xiao, X. Zhan, Z. Bian, K. Wang, X. Zhang, X. Wang, J. Li, K. Mochizuki, D. Kim, N. Kawakami et al., Observation of topological edge states in parity-time-symmetric quantum walks, Nat. Phys. 13, 1117 (2017).

[28] T. Nitsche, T. Geib, C. Stahl, L. Lorz, C. Cedzich, S. Barkhofen, R. F. Werner, and C. Silberhorn, Eigenvalue measurement of topologically protected edge states in split-step quantum walks, New J. Phys. 21, 043031 (2019).

[29] T. Schwartz, G. Bartal, S. Fishman, and M. Segev, Transport and anderson localization in disordered two-dimensional photonic lattices, Nature (London) 446, 52 (2007). 
[30] L. Levi, Y. Krivolapov, S. Fishman, and M. Segev, Hypertransport of light and stochastic acceleration by evolving disorder, Nat. Phys. 8, 912 (2012).

[31] A. Crespi, R. Osellame, R. Ramponi, V. Giovannetti, R. Fazio, L. Sansoni, F. De Nicola, F. Sciarrino, and P. Mataloni, Anderson localization of entangled photons in an integrated quantum walk, Nat. Photonics 7, 322 (2013).

[32] A. Schreiber, K. N. Cassemiro, V. Potoček, A. Gábris, I. Jex, and C. Silberhorn, Decoherence and Disorder in Quantum Walks: From Ballistic Spread to Localization, Phys. Rev. Lett. 106, 180403 (2011).

[33] A. Geraldi, A. Laneve, L. D. Bonavena, L. Sansoni, J. Ferraz, A. Fratalocchi, F. Sciarrino, Á. Cuevas, and P. Mataloni, Experimental Investigation of Superdiffusion Via Coherent Disordered Quantum Walks, Phys. Rev. Lett. 123, 140501 (2019).

[34] M. Giona and H. E. Roman, Fractional diffusion equation for transport phenomena in random media, Physica A 185, 87 (1992).

[35] Y. Yin, D. E. Katsanos, and S. N. Evangelou, Quantum walks on a random environment, Phys. Rev. A 77, 022302 (2008).

[36] A. Schreiber, K. N. Cassemiro, V. Potoček, A. Gábris, P. J. Mosley, E. Andersson, I. Jex, and C. Silberhorn, Photons
Walking the Line: A Quantum Walk with Adjustable Coin Operations, Phys. Rev. Lett. 104, 050502 (2010).

[37] T. Nitsche, S. Barkhofen, R. Kruse, L. Sansoni, M. Štefaňák, A. Gábris, V. Potoček, T. Kiss, I. Jex, and C. Silberhorn, Probing measurement-induced effects in quantum walks via recurrence, Sci. Adv. 4, eaar6444 (2018).

[38] S. Barkhofen, L. Lorz, T. Nitsche, C. Silberhorn, and H. Schomerus, Supersymmetric Polarization Anomaly in Photonic Discrete-Time Quantum Walks, Phys. Rev. Lett. 121, 260501 (2018).

[39] A. S. Pikovsky and D. L. Shepelyansky, Destruction of Anderson Localization by a Weak Nonlinearity, Phys. Rev. Lett. 100, 094101 (2008)

[40] I. Vakulchyk, M. V. Fistul, and S. Flach, Wave Packet Spreading with Disordered Nonlinear Discrete-Time Quantum Walks, Phys. Rev. Lett. 122, 040501 (2019).

[41] C. Navarrete-Benlloch, A. Pérez, and E. Roldán, Nonlinear optical Galton board, Phys. Rev. A 75, 062333 (2007).

[42] T. Nitsche, S. De, S. Barkhofen, E. Meyer-Scott, J. Tiedau, J. Sperling, A. Gábris, I. Jex, and C. Silberhorn, Local Versus Global Two-Photon Interference in Quantum Networks, Phys. Rev. Lett. 125, 213604 (2020). 